\title{
KONTROLLE IN DER VOLKSREPUBLIK CHINA
}

\author{
Von OsKar Weggel
}

\section{Der systematische Stellenwert der Kontrolle im chinesischen Verfassungssystem}

Seit Montesquieus Mißverständnis der englischen Verfassung ist die Drei-GewaltenLehre eiserner Bestandteil nahezu aller staatsrechtlichen Darstellungen im westlichen Schrifttum geworden. Diese aprioristische Betrachtungsweise hat sich merkwürdigerweise halten können, obwohl beispielsweise Institutionen wie der Rechnungshof oder der skandinavische Ombudsman im überkommenen Dreierschema von Verwaltung, Rechtsprechung und Gesetzgebung kaum unterzubringen sind.

Kein Wunder, daß die chinesische Rezeption europäischer Rechtsvorstellungen in den zwanziger Jahren, bei aller „Übersetzungstreue“, die sie sonst wahrte, den drei „westlichen“ Gewalten noch zwei „chinesische“ hinzufügte, nämlich die „Prüfungs-“ und die „Kontroll“-Gewalt ${ }^{1}$. Wahrgenommen werden die Kompetenzen, die sich aus dieser Pentastruktur ergeben, von fünf Yüans (Regierungshöfen). Der KontrollYüan, das höchste Kontrollorgan des Staates ${ }^{2}$, übt hierbei Befugnisse aus, wie sie in der Bundesrepublik Deutschland etwa den Rechnungshöfen und den Dienststrafbehörden zukommen ${ }^{3}$, wobei allerdings zu beachten bleibt, daß auf die heutige Institution noch jene konfuzianische Tradition abgefärbt hat, derzufolge die Kontrolle mehr moralische als juristische Funktionen aufweist.

War das traditionelle Dreiteilungsschema also schon für eine an westlichen Staatsbegriffen orientierte chinesische Verfassungsbewegung unannehmbar, so mußte es erst recht bei den Kommunisten auf Ablehnung stoßen. $\mathrm{Da}$ die chinesischen Kommunisten aber zumindest in den Jahren von 1949 bis 1958 weitgehend dem sowjetischen Vorbild folgten, ist es sicher nicht abwegig, einen Blick auf die sowjetischen Auffassungen zu werfen. Gerade im Hinblick auf die Sowjetunion hat Meiss$n^{4}{ }^{4}$ ein Fünf-Gewalten-Schema vorgeschlagen, das den drei herkömmlichen Funktionen noch Kontrolle und Planung hinzufügt. Ob Meissners Hypothesen zutreffen, sei dahingestellt. Für das kommunistische China jedenfalls wollen sie nicht so recht passen; denn mit bestem Willen lassen sich beispielsweise weder die Gesetzgebung 5 noch die Justiz ${ }^{6}$ als eigenständige Gewalten identifizieren. Nach alledem empfiehlt sich die wesentlich pauschalere Einteilung in Führung, Ausführung und Kontrolle. Selbst dieses vereinfachte Kategoriensystem gerät freilich ins Wanken, sobald man es z. B. auf die Armee anwendet: Führt sie oder kontrolliert sie oder führt sie nur aus? Offensichtlich lassen sich diese Fragen nicht generell beantworten; vielmehr kommt es auf die ständig sich ändernde historische Situation an. Genau genommen läßt sich eigentlich nur ein Rahmen abstecken, der die Amplituden möglicher Entwicklungen angibt. Je mehr sich das gesellschaftliche Leben "revolutioniert", je

1 Sun Yat-sen, "San min chu-i ${ }^{\star}$, The Three Principles of the Peoples, with two supplementary chapters by Chiang Kai-shek, Taipei o. J. S. $146 \mathrm{ff}$.

2 Art. 90 der Verfassung der Republik China vom 1. Januar 1947.

3 Näheres vgl, bei Oskar Weggel „Zentralregierung und Provinzverwaltung auf Taiwan“, Verfassung und Recht in Übersee 1968, S. 399.

4 Boris Meissner "Die Rechtsstellung der Kommunistischen Partei der Sowjetunion“, Jahrbuch für Ostrecht 1961 2/2, S. 7-29 (15).

5 Vgl. dazu Näheres Oskar Weggel „Die Gesetzgebung der Volksrepublik China“, in Verfassung und Recht in Übersee 1970, S. 139-166.

6 Vgl. dazu unten IV. 2 f. cc. 
mehr also vor allem die Bürokratie in den Hintergrund und der Gedanke der Selbsterziehung der Massen nach vorne rückt, um so stärker werden sich die Konturen zwischen Führung, Ausführung und Kontrolle verflüchtigen. Umgekehrt treten die einzelnen Gewalten als solche um so deutlicher hervor, je mehr die Institutionalisierung auf Kosten der Revolution zunimmt.

Mit diesen Einschränkungen soll also die Einteilung der "Gewalten“ in Führung, Ausführung und Kontrolle im folgenden beibehalten werden.

\section{Der Begriff der Kontrolle}

Gewisse Schwierigkeiten tauchen auf, wenn man den Kontrollbegriff zu operationalisieren beginnt. Von institutionalisierter Kontrolle, an die ein westlicher Beobachter sofort zu denken geneigt ist, kann in der Volksrepublik China (VRC) nämlich nur in wenigen Ausnahmefällen die Rede sein. Selbstverständlich ist auch die Rechtsfigur der Kontrolle, wie sie beispielsweise im deutschen Selbstverwaltungsrecht auftaucht, viel zu eng und damit unbrauchbar. Es empfiehlt sich vielmehr, zunächst einmal ganz allgemein an den Begriff der sozialen Kontrolle (social control) zu denken, deren Funktion es ist, unerwünschtes Verhalten zu eliminieren und dadurch die einzelnen Adressaten $\mathrm{zu}$ gesellschaftlichem Konformismus zu veranlassen. Es läßt sich nicht einwenden, daß eine solche begriffliche Ausdehnung allzu weit über das Rahmenwerk des Juristischen hinausgreift. Gerade für das sino-kommunistische Rechtsdenken ist es ja bezeichnend, daß Recht und allgemeine gesellschaftliche Normen nie so sauber voneinander geschieden werden wie in der westlichen Rechtsauffassung? ${ }^{7}$. Der so verstandene Kontrollbegriff läßt sich nach vier Richtungen hin präzisieren:

1. Nach dem Kontrollziel sind Ausführungs-, Führungs- und Massenkontrolle zu unterscheiden.

Die Ausführungskontrolle stellt darauf ab, ob die Tätigkeit der ausführenden Organe, seien es nun Staatsbehörden oder Parteigliederungen oder Massenorganisationen, an den politischen Entscheidungen und Richtlinien der Führung orientiert ist.

Die Kontrolle über die Führung hängt aufs engste mit der Massenkontrolle zusammen. Die „Massenlinie“ soll sicherstellen, daß Führung und Geführte sich gegenseitig anpassen. Es gilt, die Meinungen der Massen zu sammeln, zu konzentrieren und sie wieder in die Massen zu tragen, also einen sich unendlich spiralförmig weiter entwickelnden Prozeß einzuhalten ${ }^{8}$. Unter diesem Gesichtspunkt kann sich Kontrolle sowohl von unten nach oben wie von oben nach unten richten. Wie es heißt, soll die Führung "von den Massen lernen“ und sich "durch die Massen rückerziehen lassen“. Ein Parteiausschuß hat z. B. periodische „Ausrichtungsbewegungen der offenen Tür“ $z u$ veranstalten, um die Meinung der Massen über die einzelnen Parteimitglieder zu erfahren. Außerdem hat er über seine Tätigkeit in regelmäßigen Abständen zu berichten und die Kritik der Adressaten entgegenzunehmen. Auch empfiehlt es sich für die einzelnen Ausschußmitglieder, sich über die „Meinung der

\footnotetext{
7 Vgl. dazu Näheres Oskar Weggel „Die Gesetzgebung der Volksrepublik China“, a.a. O., S. 150, insbesondere 162 f.: Das Recht der Legisten im alten China wurde z. B. immer mehr konfuzianisiert (li contra fa); im modernen Recht wird das aus der Sowjetunion rezipierte Recht immer mehr "maoisiert“.

8 Worte des Vorsitzenden Mao Tse-tung, Peking 1967, S. 152 f.
} 
Massen" durch ausführliche Diskussion klar zu werden und außerdem regelmäßig an der Produktionsarbeit sowie am ideologischen Studium der Massen teilzunehmen'. In diesem Sinne obliegt es der Führung, „die Massen fortgesetzt zur Bewertung und Beaufsichtigung der Parteimitglieder zu mobilisieren"10. „Die Massen an Parteiversammlungen teilnehmen zu lassen und ständig Kommentare und Gegenkommentare auszutauschen, ist ein wichtiger Bestandteil von Maos Thesen über den Parteiaufbau ${ }^{11}$." Ob diesen Anforderungen auch stets genügt wird, steht auf einem anderen Blatt. Letzten Endes hängt das Ausmaß ihrer Verwirklichung vom Zustand der "Revolution“ ab. Wo Bürokratismus und „Schurigelei“ der Massen einsetzt ${ }^{12}$, ist die „Linie des Vorsitzenden Mao über den Parteiaufbau“ verlassen. Die Kontrolle richtet sich aber nicht nur von unten nach oben, sondern gleichzeitig - und in der Praxis überwiegend - von oben nach unten. Mitglieder von Parteiausschüssen oder Revolutionskomitees haben z. B. dafür zu sorgen, daß die Massen regelmäßig am Studium der Mao-Tse-tung-Ideen teilnehmen, daß sie sich Zeitungslesegruppen anschließen oder an politischen Diskussionen beteiligen.

Erwähnt sei an dieser Stelle noch, daß die gegenseitige Kontrolle der Machtträger im Sinne der Machtbalance (vgl. z. B. die Balance zwischen dem Parteisekretär, dem ZK und dem Politbüro im VIII. ZK) nicht zur Kontrolle rechnet, sondern dem Problemkreis der „Entscheidungsbildung“ angehört.

2. Nach den Kontrollmitteln ist zwischen „innerer“ und „äußerer“ Kontrolle zu unterscheiden ${ }^{13}$.

a) Äußere Kontrolle ist ein Überwachungssystem, das durch einen laufbahnmäßig organisierten und institutionell verselbständigten Apparat getragen wird. In der Sow jetunion war z. B. die stalinistische Geheimpolizei der klassische Ausdruck eines solchen Systems der äußeren Kontrolle. Anfang der fünfziger Jahre spielte in der VR China auch ein wirtschaftlicher Kontrollapparat eine nicht unwesentliche Rolle, dessen Aufgabe es war, im Namen der Pekinger Zentralregierung die Kao-KangRegierung in der Mandschurei zu überwachen. Dieser Apparat ist unter der Bezeichnung „Harbin"-System bekannt geworden ${ }^{14}$.

b) Innere Kontrolle, die in den Bezugsrahmen der "Sozialkontrolle“ gehört, ist ein System prophylaktischer Überwachung durch permanente Indoktrination. Sie zielt also hauptsächlich auf „richtiges Denken“ ab. Vor allem Techniken der Massenkommunikation sind hier nützlich, wie man sich ja überhaupt fragen kann, ob der Integrationsprozeß in der Volksrepublik China nicht ungleich stärker durch Massenkommunikation als durch die Administrative, also durch Machtanwendung, gesteuert wird. $\mathrm{Zu}$ den Mitteln der Massenkommunikation, die im China Mao Tsetungs eine bedeutende Rolle spielen, gehören etwa Plakat- und Briefaktionen, intensive Gruppenarbeit, Kampfversammlungen („Versammlungen zur Kritik und Ver-

\footnotetext{
9 Vgl. dazu Summary of World Broadcasts Part III The Far East, Reading/Engl., hrsg. von der BBC (fortan ${ }_{n} \mathrm{SWB}^{*}$ ), 3581 B II 10.

10 Peking Rundschau $1971 \mathrm{Nr}$. 29, S. 9. Research Service, hrsg. vom Union Research Institute Hong Kong (fortan "URS“), Vol. 57 (1969), S. 108.

$12 \mathrm{Vgl}$. in "diesem' Sinne auch Rosa Luxemburg „Organisationsfragen der russischen Sozialdemokratie ${ }^{*}$, abgedruckt in Rosa Luxemburg Schriften zur Theorie der Spontaneität, Reinbek bei Hamburg $1970, \mathrm{~S}$. $78 \mathrm{f}$.

$13 \mathrm{Zu}$ den Begriffen innere und äußere Kontrolle vgl. Franz Schurman, „Ideology and O rganization in Communist China “ Berkeley and Los Angeles, 1968, 2 Ed., S. 313, 339 ff.

14 Näheres hierzu ebd.. 327 ff.
} 
urteilung“), Aktivistenkongresse, sogen. „4-5-Gut-Kongresse“, „Mao Tse-tung-Studienkurse", Wandzeitungen, Losungen, Konsum von revolutionären Opern, Balletten, Konzerten und ähnlichen „Musterstücken“, Lektüre der „Worte des Vorsitzenden Mao Tse-tung“, „Bereden“ im Sinne des traditionellen „Shang-liang“, Gespräche unter vier Augen $^{15}$ und eine Fülle verschiedenartiger Konferenzen, bei denen nicht nur sachliche, sondern auch "revolutionäre" Erfahrungen ausgetauscht werden, z. B. Finanz-, Handels-, Baumwoll-, Maschinenbau-, Journalisten-, Erziehungs-, Gesundheits- und "Lernen von Tachai“-Konferenzen ${ }^{15}$. Diese Veranstaltungen finden in Form von Seminaren, aber auch in der Gestalt von sogen. „Radio- und Telefonkonferenzen" statt. Manchmal wird bei solchen Veranstaltungen den Teilnehmern auch der "Eid“ abgenommen, daß sie "mehr arbeiten und noch größere Siege in der Revolution erringen" wollen ${ }^{16}$. Worauf es bei all diesen Kommunikationsimpulsen ankommt, drückt Lin Piao folgendermaßen aus: „Wir müssen uns selbst als einen Teil der Kraft der Revolution betrachten und uns selbst zugleich ständig zum Ziel der Revolution machen. Wir müssen uns selbst in der Revolution revolutionieren ..."17. Die Umgestaltung der Welt schließt die Verwirklichung folgender Aufgaben ein: die Umgestaltung der objektiven Welt sowie die Umgestaltung der eigenen subjektiven Welt ${ }^{18}$. Neben der Sozialisierung der Produktionsmittel kommt es also vor allem auf die Sozialisierung des Denkens an.

Innere Kontrolle, wie sie in China geübt wird, ist nach alledem eher positiv (Überredung, Suggestion, Erziehung) als negativ (Drohungen, Zwangsmaßnahmen, Strafen) ${ }^{19}$, mehr informell (Beifall, Auszeichnung, Wettbewerb, Erhebung zum "Modell“)20 als formell (Gesetz, Satzungen), obgleich die sino-kommunistischen Mechanismen so sehr ritualisiert sind, daß schon fast wieder von formeller Kontrolle die Rede sein könnte. - Innere Kontrolle zielt ferner auf „bewußte Mitarbeit" ab: Nicht zufällig taucht der Ausdruck „chüeh-wu“ („Bewußtsein“) fast in jedem wichtigen Dokument auf. Unbewußt passives, wenngleich konformes Verhalten wird als ungenügend angesehen: „Die Umwandlung der Weltanschauung bedeutet, sich zu einem konsequenten Anhänger des dialektischen und historischen Materialismus und zu einem standhaften Kämpfer für die Weiterführung der Revolution unter der Diktatur des Proletariats umzuerziehen, indem man sein Denken in Ubereinstimmung mit dem Entwicklungsgesetz der objektiven Welt und mit den Forderungen der Entwicklung der revolutionären Situation bringt und die konkrete, dem historischen Verlauf entsprechende Einheit des Subjektiven mit dem Objektiven, der Erkenntnis mit der Praxis erreicht ${ }^{20 a}$."

Da Erkenntnis nicht a priori, sondern nur aus der revolutionären Praxis der Volksmassen - d. h. aus den „drei großen revolutionären Bewegungen“ (Klassenkampf, Produktionskampf, wissenschaftliches Experiment) - entsteht, ist innere

15 Näheres hierzu hat der Autor ausgeführt in „Massenkommunikation in der Volksrepublik China“, Bd. 38 der ,Mitteilungen des Instituts für Asienkunde Hamburg “, Hamburg 1970, S. $47 \mathrm{ff}$.

15a Nachweise im einzelnen bei Oskar Weggel „Die Partei als Widersacher der Revolutionskomitees“, Bd. 34 der "Mitteilungen des Instituts für Asienkunde Hamburg, Hamburg 1970, S. $69 \mathrm{f}$.

16 Daher die Bezeichnung "Eidabnahmeversammlung“ (hsüan shih ta hui) vgl. etwa SWB 3275 B II 2.

17 Peking Rundschau 1971 "Nr. 25, S. 11.

18 Ebenda S. 11 und Mao Tse-tung „Uber die Praxis“ in „Ausgewählte Werke“ Bd. I Peking 1968, S. 347 ff., insbes. $358 \mathrm{ff}$.

19 Zum Verhältnis zwischen „materiellen Anreizen“, Strafen, Erziehen usw. vgl. Weggel, „Massenkommunikation" a.a.O. $15 \mathrm{ff}$.

20 Zur Erziehung durch Modelle vgl. ebenda S. 31 f. Die Aktivistenkongresse und 4-5-Gut-Kongresse werden nur von solchen Vertretern beschickt, die sich durch besonders vorbildliches Verhalten hervorgetan haben. Vgl. z. B. SWB 3305 B 12 ff., 3325 B II 8; 3338 B II 2; zur Auszeichnung von "4-Gut-Einheiten" und 4-Gut-Einzelkämpfern “ vgl. SWB 3250 B II 19; 3267 B II 11; zum Thema Wettbewerb vgl. etwa SWB 3193 B II 8 (Eisen- und Stahlwerke des ganzen Landes folgen dem Wettbewerbsaufruf des Pekinger Eisen- und Stahlwerks, SWB 3194 B III 1. 
Kontrolle vor allem durch manuelle Arbeit (Hinunterschicken in die Dörfer) und durch häufige Teilnahme an „Versammlungen der Kritik und Verurteilung“ sowie durch Selbstkritik gewährleistet. Daher auch die Formel: „Ein Messer wird rostig, wenn man es nicht wetzt, das Wasser wird faul, wenn es stillsteht und ein Funktionär wird revisionistisch, wenn er nicht an körperlicher Arbeit teilnimmt ${ }^{21}$. Ganz in diesem Sinne werden sogen. „Kaderschulen des 7. Mai“ eingerichtet, die Bestandteile des "fundamentalen Prinzips der Verwaltungsreform sind und die dem Kampf gegen den Bürokratismus dienen“ 22 .

So kommt es auch, daß die gesamte Geschichte der Volksrepublik China seit ihrem Bestehen eine Geschichte permanenter Kampagnen und Überzeugungsfeldzüge an den verschiedenen "Fronten“ der Kultur, der Wirtschaft und der Verwaltung ist. Zumindest seit der Kulturrevolution teilen die Kommunisten selbst ihre Geschichte fast nur noch nach Kampagnen ein. Alles in allem sucht das kommunistische Regime also mit einem Minimum an Gewalt und einem Maximum an Psychologie zurechtzukommen.

3. Nach der Kontrollzeit wird im sino-kommunistischen Sprachgebrauch zwischen Ex-ante-Kontrolle (chien-tu) und Ex-post-Kontrolle (chien-ch'a) unterschieden, wobei die Ex-ante-Kontrolle weitgehend mit der „inneren“ Kontrolle identisch ist und sich deshalb auch als „Steuerung" bezeichnen ließe.

4. Ergänzend sei noch zwischen formeller und informeller Kontrolle unterschieden, eine Einteilung, die sich z. T. mit dem Begriffspaar „äußere Kontrolle“ „innere Kontrolle“ überschneidet. Während freilich äußere Kontrolle lediglich als Korrelat zur inneren Kontrolle verstanden sein will, stellt das Kriterium „formelle Kontrolle" mehr darauf ab, ob ein Gremium seiner Kompetenzverteilung nach ausdrücklich für Kontrollzwecke geschaffen ist. Formelle Kontrolle ist demnach zwar stets auch äußere Kontrolle; dagegen muß äußere Kontrolle nicht unbedingt formell sein. Der erste Teil dieser Aussage leuchtet ohne weiteres ein, der zweite ergibt sich aus der Tatsache, daß gewisse Kontrollbefugnisse zwar formellen Kontrollorganen übertragen sind (z. B. dem Kontrollausschuß in der Satzung des VIII. Parteitags, der inzwischen abgeschafft wurde), daß im übrigen aber die Kontrollbefugnisse meist auf viele Schaltstellen der Macht verteilt sind, die nicht ausdrücklich als Kontrollorgane gelten.

Inhaltlich kann sich diese Kontrolle auf so verschiedene Bereiche wie politisches, soziales, wirtschaftliches oder technisches Verhalten erstrecken. Für das traditionelle China ließe sich die Kontrolle anhand des Bildes mehrerer um ein Individuum gelegter konzentrischer Kreise illustrieren: Den äußersten Kreis bildete die Gesetzesordnung, die den Militärdienst, steuerliche Abgaben und Sicherheitsfragen regelte; weiter innen folgten gewisse Berufsvorschriften, wie sie z. B. von Gilden ausgingen; den innersten Ring schließlich, der die stärksten $Z$ wänge ausübte, bildeten Sitten und Gebräuche, die mit Hilfe von Dorfgemeinschaften, Klan und Familie aufrechterhalten und durch den Antrieb zu permanenter Loyalitätsbezeugung internalisiert wurden. All diese verschiedenen Kreise sollen nun im China Mao Tsetungs zu einer einzigen Linie zusammenfließen: Zum „politischen Verhalten“, das durch „lebendiges Lernen und lebendige Anwendung der Mao-Tse-tun-Ideen“ defi-

21 Peking Rundschau 1971 Nr. 25, S. 11.

22 Peking Rundschau 1969 Nr. 48, S. 6, Zur Bedeutung des „7. Mai“ s. unten Anm. 87. 
niert ist: als geistige Bereitschaft also, die Richtlinien Maos auf jedem nur denkbaren Gebiet zu befolgen und die von ihm vorgeschriebenen Verhaltenschemata zu habitualisieren. Politik gilt als die "Seele“. Sie hat „das Kommando zu übernehmen“; sie drängt alle "fachmännischen", wirtschaftlichen, familiären und „humanitären“ Erwägungen in den Hintergrund ${ }^{23}$. Die maoistische Kontrollstrategie zielt also mit anderen Worten darauf ab, den 800 Millionen ein neues Selbstverständnis zu vermitteln.

Die Familie hat demzufolge, will sie sich "politisch“ richtig verhalten, nicht mehr durch patriarchalische Gewalt zu kontrollieren, sondern nur noch durch FamilienStudienkurse ${ }^{24}$ und „Familien-Kampfversammlungen“25. Der Staat andererseits braucht nur noch gegen die Klassenfeinde mit den Mitteln der Diktatur des Proletariats, also mit physischer Gewalt, vorzugehen. Nicht-antagonistische Widersprüche, d. h. „Widersprüche im Volk“ dagegen sollen formell durch die „demokratischen Methoden“ der Überzeugung und Erziehung, inhaltlich durch die Mao-Tsetung-Ideen gelöst werden. Diese ganz und gar „politisch“ eingefärbte Kontrolle soll sicherstellen, daß in einer Zeit zunehmender gesellschaftlicher Differenzierung, beruflicher Spezialisierung und Bürokratisierung das Vorbild des sozial universalen Guerilla-Kämpfers seine modellhafte Verbindlichkeit behält. Der in der Soziologie weit verbreitete Begriff der „social control“ erhält damit einen spezifischen Inhalt. Hand in Hand mit der Entinsituationalisierung der Kontrollfunktionen ergehen ständig Aufrufe zur Verwaltungsvereinfachung, zur Änderung schwerfälliger und „unvernünftiger" Regelungen und zur Freisetzung von Büroangestellten für den Einsatz auf unterer Ebene.

Faßt man diese vier Punkte zusammen, so ergibt sich folgende Definition: Kontrolle in der VR China ist ein System der Beaufsichtigung, dessen Zweck es ist, ex ante oder aber ex post die Ausführung von Weisungen und politischen Richtlinien (Durchführungskontrolle), aber auch umgekehrt die Führungsorgane zu überwachen (Führungskontrolle), wobei Indoktrinisierungsmaßnahmen im weitesten Sinne (innere Kontrolle) oder aber organisatorisch verselbständigte Apparate eingesetzt werden (äußere Kontrolle), die ihrerseits entweder ausdrücklich für Kontrollzwecke vorgesehen sind (formelle Kontrolle) oder aber Kontrollbefugnisse implizit neben anderen Aufgaben wahrnehmen (informelle Kontrolle).

\section{Die Entwicklung der Kontrolle in der Volksrepublik China}

Die Kontrollpolitik ist sich in der Volksrepublik China nicht immer gleich geblieben. Herrschte in der ersten Hälfte der fünfziger Jahre noch vielfach das Prinzip der äußeren Kontrolle, das zumeist mit Ausführungs- und Ex-post-Kontrolle identisch war, so verlagerte sich das Schwergewicht seit dem 8. Parteitag (1956) auf die innere Kontrolle ${ }^{26}$. In diesem Wandel klang das alte Grundthema der "Massenlinie“ wieder an. Kritik und Selbstkritik, Plakat- und Briefaktionen, hsia-fang ${ }^{26 a}$ und intensive Gruppenarbeit sollten fortan den formalen und bürokratisierten Inspek-

23 Näheres Weggel, „Die Partei als Widersacher der Revolutionskomitees“, a.a.O., S. 76 ff. Vgl. hier z. B. China News Analysis, Hong Kong (fortan CNA) Nr. 423, 505, 849.

24 Näheres Weggel, Massenkommunikation, a.a.O., $134 \mathrm{f}$.

25 Vgl. z. B. die sogen. „die ganze Familie-rot-Bewegung “, SWB 3198 B II 6

26 Vgl. Schurmann a.a.O., S. $339 \mathrm{ff}$.

26a "Hsia fang ${ }^{\alpha}$ ist ein Ausdrudk, der das „Hinunterschicken ${ }^{\alpha}$ zu körperlicher Arbeit zum Ausdrudk bringt. 
tionsmechanismus überflüssig machen. Damit aber war bereits die zweite Verschiebung gegeben: Während nämlich vorher nur das äußere Handeln vom Scheinwerfer der Kontrolle abgetastet wurde, sollte nunmehr der innere Mensch zurechtgerückt und in seinem Denken gesteuert werden. Die Kontrollreform von 1956 baute die Massenlinie noch nicht bis zu ihrer Vollendung aus; betraf sie doch nur die Parteiausschüsse, während die breite Masse des Volkes weiterhin Objekt der äußeren Kontrolle blieb. Erst die Kulturrevolution fügte den Schlußstein in das Gewölbe, indem sie die Kontrollgewalt im Bereich der unzähligen Einheiten den Revolutionskomitees überantwortete ${ }^{27}$. Von dieser Entwicklung her gesehen ließe sich also eine idealtypische Verschiebung von der Staatskontrolle über die Parteikontrolle zur Massenselbstkontrolle und damit eine Versöhnung der Wirklichkeit mit der Idee der Massenlinie feststellen. Freilich gelang es dem Militär, nach und nach über den Mechanismus der Drittelparität in den einzelnen Revolutionskomitees und auch in den seit Anfang 1969 wieder entstehenden neuen Parteiausschüssen so starken Einfluß zu gewinnen, daß die Massenselbstkontrolle praktisch nur noch Postulat blieb. Die Fiktion nämlich, daß Massen und Militärs identisch seien und Militärkontrolle daher praktisch der Massenselbstkontrolle nichts anhaben könne, ist unhaltbar ${ }^{28}$.

\section{Kontrollorgane und Kontrollinstrumente}

Aufgrund des Führungsmonopols der Partei sowie des Instrumentalcharakters von Staat und Massenorganisationen hat die Partei grundsätzlich die politische Entscheidung zu treffen; Staat und Massenorganisationen haben sie durchzuführen, wobei die Durchführung wiederum überwiegend von der Partei kontrolliert wird. Diese Verflochtenheit macht es schwierig, spezifische Kontrollformen aus dem Gesamtmechanismus auszusondern. Trotzdem soll hier versucht werden, Parteikontrolle (einschl. der Kontrolle durch Massenorganisationen), Staatskontrolle und Militärkontrolle gesondert darzustellen.

\section{Parteikontrolle: Die Partei kontrolliert in dreifacher Weise}

a) Im Zuständigkeitsbereich sämtlicher Administrativorgane bestehen Parteiausschüsse, so daß man von einer Art Doppelregierung reden könnte. Je höher die Schlüsselpositionen werden, um so dichter sind sie - ebenso wie in der Sowjetunion - mit Parteimitgliedern besetzt, die weniger "fachlich" als "organisatorisch“ orientiert sind und die weniger auf administrative Erledigung als vielmehr auf Erfüllung ihrer Aufgaben im „Kampagnenstil“ achten. Die enge Verflochtenheit von Staats- und Parteiorganen kommt auch dadurch zum Ausdruck, daß wichtige Direktiven bisweilen von ihnen gemeinsam herausgegeben werden, besonders häufig vom ZK und vom Staatsrat ${ }^{29}$.

\footnotetext{
27 Im einzelnen vgl. hierzu Weggel, Oskar, „Die chinesischen Revolutionskomitees“, Bd. 25 der Mitteilungen des Instituts für Asienkunde Hamburg, Hamburg 1968, S. $96 \mathrm{f}$ 28 Näheres vgl. dazu unten IV. 3.

29 Weggel, Gesetzgebung a.a.O., S. 144, 147.
} 
b) Ferner fungieren innerhalb der einzelnen kulturellen, pädagogischen und wirtschaftlichen Einheiten (z. B. Schulen, Fabriken, landwirtschaftliche Produktionsbrigaden) Grundorganisationen, die je nach Mitgliederzahl als „Zellen“, „Hauptzellen“ oder "Basisausschüsse" firmieren ${ }^{30}$.

c) Ihre eigenen Mitglieder überwachte die Partei bis zur Kulturrevolution durch sogen. Kontrollausschüsse. Diese Form der äußeren Kontrolle war durch Art. 52-54 der „liu-istischen“ Parteisatzung von 1956 vorgeschrieben. Der damalige Kontrollapparat war von der Zentrale bis hinunter zur Kreisebene den Parteiausschüssen zugeordnet und hatte die Aufgabe, Verletzungen der Parteisatzung durch Parteimitglieder festzustellen und den jeweiligen Parteiausschüssen disziplinarische Maßnahmen zu empfehlen. Fünf Disziplinarstrafen standen im wesentlichen zur Verfügung: Erziehung durch Kritik, Warnungen, Eintragung in das persönliche Führungsregister, Einräumung einer Bewährungsfrist und Ausstoßung aus der Partei $^{31}$. Waren die Kontrollausschüsse ${ }^{32}$ in der Parteisatzung von 1945 nur als Ad-hoc-Organisationen für die Parteiausschüsse auf der jeweiligen Ebeene vorgesehen, so fungierten sie seit 1956 als institutionalisierte Agenturen der Parteihierarchie $^{33}$. In der "maoistischen“ Parteisatzung von 1969 sind die formellen Kontrollorgane bezeichnenderweise weggefallen. Nun herrscht weitgehend innere Kontrolle, die durch eine Reihe von Disziplinierungsinstrumenten sichergestellt wird, z. B. durch die obligate regelmäßige Teilnahme am "Parteileben“ (Diskussionen, Kritik und Selbstkritik, "Austausch von Erfahrungen“), tägliches politisches Studium in sogen. „kleinen Studiengruppen"34, deren Dauer und Intensität vor allem bei jeder neuen Kampagne spürbar zunimmt, durch sorgfältig geführte Personalakten ${ }^{35}$ und Verhaltensbewertungen, die am Ende jeden Jahres oder am Ende einer Kampagne getroffen werden ${ }^{36}$.

Weitere Kontrollinstrumente sind die sogen. „Ausrichtungsbewegungen “, die den drei großen Arbeitsstilen (Verbundenheit zwischen Kadern und Massen, Verbundenheit von Theorie und Praxis, Kritik und Selbstkritik) wieder zu ihrem Recht verhelfen und mit bürokratischen Attitüden ins Gericht gehen sollen. Gerade im Zusammenhang mit diesen Kampagnen wird vom Parteiausschuß jeweils ein eigener Ad-hoc-Stab geschaffen, der vor allem Kampfveranstaltungen durchführt ${ }^{37}$. Nicht zuletzt aber ist es die immer wieder betonte körperliche Mitarbeit unter den Massen, die dem Kampf gegen den Revisionismus und damit der „lebendigen Verwirklichung der Ideen des Vorsitzenden Mao“ zu dienen hat.

\footnotetext{
30 Nach Art. 48 der Parteisatzung von 1956 war in einer Einheit, in der 100 oder mehr Parteimitglieder wirkten, ein sogen. "Komitee der Grundeinheit" $z$ u bilden (dii-tseng tang wei-yüan-hui), bei 50 oder mehr Parteimitgliedern war eine sogen. ${ }_{n}$ Hauptzelle ${ }^{\alpha}$ (tsung chi pu wei-yüan-hui) und bei weniger als 50 Mitgliedern eine Zelle einzurichten (chi pu wei-yüan-hui). In der neuen Parteisatzung von 1969 befindet sich keine ähnliche zahlenmäßige Begrenzung, obwohl die drei Grundorganisationen als soldhe ausdrücklich aufgeführt sind.

31 Barnett, A. Doak Cadres, Bureaucracy and Political Power in Communist China*, New York and London 1967, S. $145^{n} \mathrm{f}$. Verfehlungen von Parteimitgliedern wurden meist von der Partei selbst geahndet und nur selten der Justiz überantwortet, vgl. ebenda $S$. $144 \mathrm{f}$.

32 Chien-ch'a wei-yüan-hui.

33 Tang, Peter S. H., und Joan M. Maloney "Communist China: The Domestic Scene 1949-1967", South Orange/N. J. 1967, S. 173.

34 Hsüeh-ksi hsiao tsu.

35 "Tang-an", die von der Organisationsabteilung des Parteiausschusses geführt wurden; sie wurden während "Tang-an", die von der Organisationsabteilung des Parteiausschusses geführt wurden; sie wurden wahrend
der Kulturrevolution besonders angegriffen. Ob die Praxis sorgfältiger Personalaktenführung seitdem wiederaufgelebt ist, kann nicht festgestellt werden.

36 Hierzu ausführlich Barnett a.a.O., S. 166 ff.

37 Ebenda, S. 107 f.
} 
Zu den Mitteln äußerer Kontrolle, wie sie im Zusammenhang mit der Partei gerade während der einflußreichsten Wirkungsjahre Liu Shao-ch'is gang und gäbe waren, gehörten auch die sogen. Arbeitsgruppen ${ }^{33}$, die von den örtlichen Parteiausschüssen in die Universitäten, Schulen, Fabriken und dergl. entsandt wurden, um dort auf Einhaltung der Parteilinie zu achten. Vor allem zu Beginn der Kulturrevolution wurden solche Gruppen häufig eingesetzt, um im Namen der Partei gegen die neu entstandenen parteifeindlichen Rotgardisten und „Roten Rebellen“ vorzugehen ${ }^{39}$. Mit der Kulturrevolution begann das große Sterben dieser „Arbeitsgruppen“. Bezeichnend hierfür war z. B. ein Beschluß des damals gerade neu gebildeten Pekinger Stadtparteiausschusses vom 28. Juli 1966, demzufolge im Interesse einer besseren Massenmobilisierung und einer Erhöhung der „revolutionären Begeisterung und des revolutionären Selbstbewußtseins" die Arbeitsgruppen an den Universitäten, Colleges und höheren Schulen aufgelöst werden sollten ${ }^{40}$. „Versammlungen der Kritik und Verurteilung" sowie Wandzeitungen bestimmten von $\mathrm{da}$ an das Leben in den Schulen. Auch hier zeigte sich deutlich die Hinwendung von der äußeren zur inneren Kontrolle, die sich in dem Maße beschleunigte, wie die „revolutionären Stürme" zunahmen.

Besonders weit gefächerte Kontrollbefugnisse stehen der Partei den „Massenorganisationen" gegenüber $\mathrm{zu}$, die in China noch ungleich wirkungsvoller als in der Sowjetunion als Transmissionen eingesetzt sind. China ist vielleicht das Land der Organisation schlechthin. Wer nicht Parteimitglied ist, gehört zumindest einer der zahlreichen Massenorganisationen an, sei es nun der Gewerkschaft, der Kommunistischen Jugendliga, dem Studentenverband, dem Bauernbund oder einer ähnlichen Gliederung. Diese zumeist „allchinesisch" 41 aufgezogenen Organisationen sind durch die Kulturrevolutionen zwar weitgehend liquidiert worden, haben aber im Zuge der allgemeinen Dezentralisierung in den zahlreichen "Versammlungen " 42 und Mao-Tse-tung-Studienkursen legitime Erben gefunden. Waren freilich die alten Massenorganisationen noch überwiegend auf äußere Kontrolle hin orientiert (die formell aufgebauten Gewerkschaftsgliederungen hatten z. B. das Recht, von der Fabrikleitung Arbeitsberichte zu verlangen und auch an den Betriebsversammlungen teilzunehmen ${ }^{43}$ ), so sind die neuen Gliederungen vor allem auf innere Kontrolle angelegt. Der grundlegende „Weg zur Revolutionierung“ wurde hierbei vor allem durch die „Weisung des Vorsitzenden Mao vom 7. Mai 1966“ aufgezeigt, nach der sämtliche Einheiten im administrativen, kulturellen, landwirtschaftlichen und industriellen Bereich sich in eine "große rote Schule“ (der Mao-Tse-tung-Ideen) verwandeln sollen ${ }^{44}$. Vor allem hier wird deutlich, daß die chinesischen Volksmassen weniger durch administrative Mittel als vielmehr durch Massenkommunikation gesteuert werden sollen. Einzelheiten zur kommunikativen Funktion der „Aktivistenkongresse“, der „4-5-Gut-Kongresse“, der „Kampfversammlungen“, der „MaoTse-tung-Studienkurse" und ähnlicher Einrichtungen hat der Autor an anderer

\footnotetext{
38 Kung-tso tsu. Sie waren ursprünglich als Gruppen für sogen. „Schlüsselexperimente* gedacht (neue Organisationsformen etc.), weiteten ihren Bereich aber immer mehr aus. Vgl. dazu etwa Barnett a.a.O., S. $139 \mathrm{f}$.

39 Vigl. dazu New China News Agency, London, (fortan NCNA), vom 2. 6. 1969, S. 16.

40 Vgl. dazu die Dokumente in "CCP Documents of the Great Proletarian Cultural Revolution 1966-1967“ hrsg. vom Union Research Institute Hong Kong, Kong Kong 1968, S. $652 \mathrm{ff}$.

41 Chüan-kuo.

42 Vertreterversammlungen der Arbeiter, der Bauern und der Rotgardisten (kung tai-hui, nung tai-hui, hung tai-hui); Aktivistenkongresse, 4-5-Gut-Kongresse usw.

43 Art. 8 der Gewerkschaftsordnung vom 29. 6. 1950. Freilich spielte auch schon die „Erziehung der Massen“ eine Rolle, wie in Art. 9 der gleichen Ordnung vermerkt ist.

44 Vgl. dazu erst jüngst wieder Volkszeitung vom 7. Mai 1971, abgedr. in Peking Rundschau 1971, Nr. 20, S. 5. $\mathrm{f}$.
} 
Stelle mitgeteilt ${ }^{45}$. Eine immer wichtigere Rolle kommt dabei den "Aktivisten“ zu, die als „Verbindungsglieder" 46 zwischen Führung und Massen dienen (also nicht der Partei angehören), die wegen ihrer Kenntnis der lokalen Verhältnisse unentbehrlich sind und die eine im Sinne von "heroischen Modellen“ vorgelebte Bereitschaft zeigen, sich im Dienste der Partei, vor allem aber Mao Tse-tungs, kämpfend einzusetzen. Aktivistenfunktionen in diesem Sinne erfüllen auch die neu gebildeten Medizingruppen und „barfüßigen Arzte“, die seit der Kulturrevolution „hinunter in die Dörfer" geschickt werden und dort nicht nur ärztliche Vorbeugungs- und Heilarbeit, sondern auch Propagandadienste leisten.

2. Staatskontrolle: Hier begegnen uns vertrautere Begriffe. Vieles ist freilich Fassade, da die Kontrolle - der allgemeinen Tendenz folgend - im Laufe der Jahre immer mehr entformalisiert und verinnerlicht wurde.

a) Der Nationale Volkskongreß übt dadurch gewisse Kontrollbefugnisse aus, daß er die Verfassung ändert und ihre Durchführung überwacht, die Vorsitzenden der wichtigsten Staatsorgane wählt und abberuft oder daß er z. B. Volkswirtschaftspläne sowie den Haushalt prüft und bestätigt ${ }^{47}$.

b) Der Ständige Ausschuß des Nationalen Volkskongresses kontrolliert die Arbeit des Staatsrats und der anderen zentralen Organe, hebt Verordnungen und Verfügungen des Staatsrats auf, die im Widerspruch zur Verfassung oder zu den Gesetzen stehen, ändert "nicht zweckentsprechende“ Beschlüsse der Staatsorgane auf Provinzebene $\mathrm{ab}$ und hebt sie auf, kontrolliert die Personalangelegenheiten usw. ${ }^{48}$.

c) Auch der inzwischen abgeschaffte „Vorsitzende“, d. h. der Staatspräsident ${ }^{49}$, übte vor allem auf dem Umweg über die höchste Staatskonferenz und den Nationalen Verteidigungsrat wichtige Überwachungsfunktionen aus.

d) Der Staatsrat überwacht die Durchführung der von ihm erlassenen Verordnungen und Verfügungen, kontrolliert die Arbeit der Ministerien und der örtlichen Organe der staatlichen Gewalt im ganzen Land, ändert „nicht zweckentsprechende“ Anordnungen $\mathrm{ab}$ und hat wichtige Rechte in der Personalpolitik ${ }^{\mathbf{5 0}}$.

e) Die örtlichen Volkskongresse und Revolutionskomitees sowie die Organe der Selbstverwaltung in den Minderheitengebieten gewährleisten in ihrem Verwaltungsgebiet die Einhaltung der Gesetze und der öffentlichen Ordnung ${ }^{51}$.

\footnotetext{
45 Massenkommunikation in der Volksrepublik China, a.a.O., S. $81 \mathrm{ff}$. und 3 ff. 46 Niu-tai.

47 Bisher gab es in der Volksrepublik China vier gesamtnationale „Fünfjahrespläne ${ }^{\alpha}$. Ob die beiden letzten Pläne (1966-1971 und 1971 ff.) mehr als nur empfehlende Funktion haben, ist zweifelhaft. Auch sind nähere Einzelheiten unbekannt, obwohl von diesen Plänen in öffentlichen Verlautbarungen immer wieder gesprochen wird. Zu den Funktionen vgl. im übrigen Art. 27 der Verfassung vom 20. Sept. 1954 und Art. 17 des Verfassungsentwurfs von 1970, letzterer abgedruckt in "Verfassung und Recht in Ubersee* 1971 S. $62 \mathrm{ff}$.

48 Art. 31 Verfassung 1954, Art. 18 Verfassungsentwurf 1970.

49 Der Verfassungsentwurf von 1970 enthält keine Bestimmung mehr über den „Vorsitzenden*. Allerdings gilt Mao Tse-tung als „der große Führer aller Nationalitäten im ganzen Staat “, als „Oberhaupt unseres Staates der Diktatur des Proletariats" und als "Oberbefehlshaber der gesamten Nation und aller Streitkräfte ${ }^{\alpha}$ (Art. 2 Entwurf). Damit wird eine Situation legalisiert, die seit der Kulturrevolution de facto ohnehin bestand.

50 Art. 49 Verfassung 1954, Art. 20 Entwurf 1970

51 Art. 58, 64 der Verfassung 1954 und Art. 23 Entwurf 1970.
} 
f) Vor der Kulturrevolution lagen die wichtigsten, sozusagen klassischen, Kontrollfunktionen bei der Dreiheit von Polizei, Staatsanwaltschaft und Gerichten, die im Rahmen der „Diktatur des Proletariats“ eine besonders wichtige Rolle spielten. Diese drei Organisationen waren eng mit Liu Shao-ch'i verbunden und wurden daher während der Kulturrevolution weitgehend ausgeschaltet und mit Militärs durchsetzt.

aa) Das Polizeiwesen: Das dem Staatsrat zugehörige „Ministerium für öffentliche Sicherheit"52 steuerte die Sicherheitsämter auf drei Ebenen (Provinzen, Sonderdistrikte und Kreise, die ihre Unterbüros ihrerseits bis hinunter zu den Produktionsbrigaden eingerichtet hatten $)^{53}$. Zu den Aufgaben der Sicherheitsämter gehörte es, für öffentliche Sicherheit und Ordnung zu sorgen ${ }^{54}$ und dabei vor allem Verbrechen zu bekämpfen, den Verkehr zu überwachen, Waffenkontrolle auszuüben, die Aktivitäten zur „Erziehung durch Arbeit“ zu überwachen, Reisegenehmigungen zu erteilen, über die Stimmung der Bevölkerung zu berichten, für Volkszählung sowie Statistik zu sorgen und vor allem die sogen. „fünf schlechten Elemente“55 zu kontrollieren. Die ständige Überwachung dieser „Elemente“, zu denen ehemalige Großgrundbesitzer, reiche Bauern, Konterrevolutionäre, Rechtsabweichler und andere wenig genau definierte „böse Elemente“ gehörten, war eine der Hauptobliegenheiten der Sicherheitsämter. Angehörige dieser fünf Gruppen waren formell als solche eingestuft. So kommt es, daß Grundbesitzer und „Reiche Bauern“, die ihr Vermögen längst eingebüßt haben, auch heute noch amtlich als "Großgrundbesitzer" und "Reiche Bauern" bezeichnet werden. Diese "politischen Parias", die etwa 1 Prozent der Bevölkerung ausmachen, unterlagen scharfen Maßnahmen bei der „Erziehung durch Arbeit“, hatten regelmäßig beim lokalen Amt für öffentliche Sicherheit Rechenschaft über ihr Verhalten abzulegen und dienten im übrigen als willkommene Ziele jener öffentlichen Kritik, die vor allem während der immer wieder anlaufenden Kampagnen ausgelöst wird. Theoretisch können Personen, die den „fünf bösen Elementen“ zugerechnet werden, zwar nach langem Wohlverhalten beim örtlichen Amt für öffentliche Sicherheit eine formale Redefinition ihres Klassenstandes beantragen. Doch wird solchen Anträgen erfahrungsgemäß nur in seltenen Fällen stattgegeben ${ }^{56}$. Außer den Behörden für öffentliche Sicherheit

\footnotetext{
52 Kung-an-pu.

53 Der Sicherheitsapparat ist in drei Stufen aufgebaut: Provinzebene: sogen. „Sicherheitsämter" (kung-an-t'ing); Sonderdistriktsebene: sogen. Sicherheitsstationen" (kung-an chu); Kreis-(Stadt-)Ebene: sogen. „Sicherheitsbüros “ (kung-an chü). Die Sicherheitsbehörden werden von den Volkskongressen ihrer jeweiligen Ebene errichtet und kontrolliert. Obwohl sie demnach eigentlich administrative Organe sind, galten sie zumindest bis zur Kulturrevolution - im allgemeinen doch als wichtigstes Instrument der lokalen Parteiorganisationen, um äußere Kontrolle über die Bevölkerung zu erlangen (Barnett a.a.O. 220). Nicht von ungefähr gehörte daher ein besonders hoher Anteil des Personals der Partei oder der parteinahen Kommunistischen Jugendliga an. Ein Teil des Personals trug Uniform und war bewaffnet (Barnett a.a.O. 221). Es war u. a. für das Gefängniswesen und für „Reform durch Arbeits-Gruppen “ zuständig (Barnett a.a.O. 225). - Die Sicherheitsbüros in den Kreisen und Städten errichteten Sicherheitsunterbüros (kung-an fenchü). Solche Unterbüros gibt es auch in den Volkskommunen. Eng mit den Sicherheitsbüros ist die örtliche Miliz verbunden (Barnett a.a.O. 390-92). Daneben gibt es - oder gab es zumindest bis zur Kulturrevolution - spezielle Sicherheitsämter in Form von Hafenpolizei, Eisenbahnpolizei und Fabrikpolizei (Art. 7 der Organisationsbestimmungen über die öffentlichen Sicherheitsunterbüros vom 31. Dez. 1954, abgedruckt in Theodore H. E. Chen, "The Chinese Communist Regime, Documents and Commentary" Eedon 1967 S 113 f. Gemäß A t 2 dieser Organisationsbestimmungen aren die Unterbüros für öffentliche Sicherheit zuständig für Aufrechterhaltung der Gesetze über öffentliche Sicherheit und soziale Ordnung, für "Unterdrückung von Sabotageakten, von Konterrevolutionären, die flagrante delicto ertappt nung, für "Unterdrückung von Sabotageakten, von Konterrevolutionären, die flagrante delicto ertappt Kontrolle über Theater, Kinos, Hotels usw., für Beteiligung an Wohlfahrtsarbeiten für die Bevölkerung und bezeichnenderweise auch für die „Ausführung von Propaganda unter der Bevölkerung in allen Fragen. welche erhöhte revolutionäre Wachsamkeit, Gesetzesbeachtung, Beachtung der öffentlichen Ordnung und Respektierung der öffentlichen Moral angehen “.

54 Vgl. dazu auch Fußnote 53.

55 Wu lei fen-tzu.

$56 \mathrm{Vgl}$. dazu im einzelnen Barnett a.a.O. $231 \mathrm{ff}$.
} 
existierten Sicherheitsgruppen von etwa 250000 Mann, die militärisch organisiert und der Armee unterstellt waren. Die Armee hielt sich im Hintergrund, während die Sicherheitsorgane ihre Routinearbeit leisteten. Darüber hinaus bedienten sich die Sicherheitsämter - zumindest bis zur Kulturrevolution - eines Geheimpolizeinetzes, das insbesondere der „Unterdrückung von Konterrevolutionären“ diente $^{57}$.

Durch die Kulturrevolution wurde der gesamte Sicherheitsapparat zerstört oder von der Volksbefreiungsarmee bzw. deren „Militärkontrollausschüssen“ übernommen und seine Funktionäre in sogen. „7.-Mai-Schulen" zur Umerziehung geschickt. Die Sicherheitskompetenzen gingen auf die Revolutionskomitees über, die nach und nach ein buntgewebtes Netz von Sicherheitsorganisationen aufbauten. Aus Shanghai liegen z. B. Meldungen über sogen. Nachbarschafts-Sicherheitstruppen vor. Diese Einrichtung hängt mit gewissen Eigenarten der Städteorganisation zusammen: Seit 1958 hatten die Kommunisten versucht, städtische Kommunen einzuführen ${ }^{53}$. Während solche Kommunen, soweit sie sich um Fabriken oder um Verwaltungsbehörden bzw. Schulen herauskristallisierten, weitgehend im Experimentierstadium steckenblieben, wurden die auf dem Straßenprinzip aufgebauten Nachbarschaften zu einer zukunftsträchtigen - weil der Dezentralisierung, Kontrolle und Autarkisierung am besten - dienenden Einrichtung, die vor allem in Shanghai auch heute noch am Leben ist. Als Muster darf die Kuang-chien Lane im X-Distrikt in Shanghai gelten, bestehend aus 1164 Familien (5578 Personen, darunter 1437 Arbeiter und 108 „Veteranen"), die für Kleinindustrien (Nachbarschaftsunternehmungen) mobilisiert werden und gemeinsam öffentliche Einrichtungen (Speisehallen, Schulen usw.) betreiben. Die Lane hat ihr eigenes Revolutionskomitee und unterhält einen militärisch organisierten "Mao-Tse-tung-Propagandatrupp“ (61 Mitglieder), der Parteiaufgaben wahrnimmt (äußere Kontrolle), „Mao-Tse-tung-Studienkurse“ betreibt und dabei u. a. Jugendliche für den Einsatz auf dem Lande mobilisiert ${ }^{59}$. Neben den in diesem Zusammenhang wirkenden „Nachbarschafts-Sicherheitstruplen“ ist auch von "Truppen des gewaltlosen Angriffs und der gewaltsamen Verteidigung" die Rede. In Canton sind es "Arbeiter-Schutzkorps“, in Kianghsi bewaffnete Kommandos der "revolutionären Arbeiter" usw. Ihre Aufgaben sind polizeilicher Natur. Sie überwachen den Straßenverkehr, schützen Brücken und andere strategisch wichtige Punkte, verfolgen kriminelle Delikte und sind auch zur Festnahme berechtigt. Sicherheitsgruppen dieser Art, die sich im wahrsten Sinne des Wortes als „Volkspolizisten“ bezeichnen können, sind meist nur durch Armbinden ausgewiesen. Sie sind entweder unbewaffnet oder tragen hölzerne Schlagstöcke. Im Sicherheitsdienst sind bisweilen auch Kinder tätig, besonders Mitglieder der sogen. „kleinen roten Soldaten", die als Straßenpatrouillen eingesetzt werden.

In den Fabriken nehmen insbesondere seit Oktober 1969, als ökonomische Fragen wieder in den Vordergrund $\mathrm{zu}$ treten begannen, sogen. "Wirtschaftskontrollausschüsse der Arbeiter"60 die Kontrolle über Buchhaltung und Finanzgebaren wahr. Organisatorisch gehen diese Kontrollausschüsse ${ }^{61}$ aus den Revolutionskomitees der

57 Ebda. 222, 226 f. Im übrigen vgl. auch Tang-Maloney a.a.O. $302 \mathrm{ff}$.

$58 \mathrm{Zu}$ den Phasen dieser Entwicklung vgl. im einzelnen Salaff, Janet "The Urban Communes and Anti-City Experiments in Communist China“, China Quarterly Nr. 29, S. 82-109 (84-89); vgl. ferner TangMaloney a.a.O. S. $388 \mathrm{ff}$

59 Radio Shanghai in SWB 3209 II $12 \mathrm{ff}$; vgl. auch SWB 3219 B 14 f.; URS Vol. 57 Nr. 4 (35 ff.).

$60 \mathrm{Kung}-$ jen ching-chi chien-tu wei-yüan-hui.

61 Manchmal helfen bei der Bildung solcher Kontrollausschüsse auch Armeegruppen zur Unterstützung der Linken mit (SWB 3455 B II 3). 
jeweiligen Fabriken hervor. Ihre Aufgabe ist es, dem Revolutionskomitee bei der Ubberwachung der Produktion, des Rohmaterialverbrauchs (u. a. Kontrolle der Lagerhäuser), der Arbeitsstundennormen sowie der Fabrikfinanzen zu helfen und darauf zu achten, daß durch erhöhte Sparsamkeit die Autarkie der einzelnen Einheiten gewährleistet und damit der Staatshaushalt vor Anforderungen geschützt wird ${ }^{62}$. Die Kontrollausschüsse sind nach offizieller Darstellung ein lebendiger Ausdruck der Teilnahme des Arbeiterstamms an der Fabrikverwaltung ${ }^{63}$.

Es ist bezeichnend für diese Kontrollausschüsse, daß die Kontrollmaßstäbe - anders als bei den sowjetischen Komitees für Arbeiter- und Bauerninspektion - nicht in erster Linie Zweckmäßigkeit und Wirtschaftlichkeit sind ${ }^{64}$, sondern "politische Richtigkeit“. Dies ist nicht weiter verwunderlich, wenn man bedenkt, daß die Mitglieder der Ausschüsse den Kontrollaufgaben vom fachlichen Standpunkt aus häufig nicht gewachsen zu sein scheinen ${ }^{65}$. Je mehr freilich die Auswirkungen der Kulturrevolution $\mathrm{zu}$ verblassen beginnen und je stärker wieder ökonomische Gesichtspunkte in den Vordergrund treten, um so mehr dürfte das "politische Element" wieder durch „fachmännische“ Gesichtspunkte verdrängt werden. Vorerst bleibt allerdings ein "politischer Arbeitsstil“ noch vorherrschend, wie auch die derzeitigen Kontrollmittel beweisen: „Kampfversammlungen“ gegen unzuverlässige Fabrikarbeiter gehören nämlich nach wie vor zu den wirksamsten Disziplinierungsinstrumenten. Durchaus "politisch" ist auch der Kriterienkatalog, dessen sich z. B. die Kontrollausschüsse in den Industriebetrieben der Stadt Tientsin bedienen. Dieser Katalog ist mit dem typisch chinesischen Namen „Ein Überblick, zwei Vergleiche und drei Nachprüfungen" versehen. Beim "Überblick“ ist festzustellen, wie hoch der durch die Verschwendung entstandene Schaden ist. Bei den „zwei Vergleichen“ geht es darum, die Leistungen der eigenen Einheit mit denen fortgeschrittener Einheiten sowie mit früheren Erfolgen zu vergleichen. Die „drei Nachprüfungen“ sind auf "Sabotage der Klassenfeinde", auf verschwenderische Verbrechen der „Kapitalisten"66 sowie auf die Bereitschaft der Arbeiterklasse zum Klassenkampf $\mathrm{zu}$ richten ${ }^{67}$. Bisweilen erlassen die Kontrollausschüsse - offensichtlich in Zusammenarbeit mit dem Fabrik-Revolutionskomitee - auch Satzungen und andere betriebsinterne Regelungen ${ }^{63}$. Zusammengesetzt sind die Kontrollausschüsse aus Arbeitern (häufig auch Arbeiterveteranen), Mitgliedern des Fabrik-Revolutionskomitees und geschultem Finanzpersonal ${ }^{70}$.

"Arbeiterkontrollausschüsse" sind nicht die einzigen Organisationen, die Überwachungsaufgaben im Fabrikbetrieb wahrnehmen. Auch „wirtschaftliche Beratungsausschüsse", an denen neben Fabrikarbeitern auch Armeeangehörige teilnehmen und die Kampagnen zum „Kampf und zur Kritik“ durchführen"1, sowie

\footnotetext{
62 Wie sehr gerade der zuletzt genannte Gesichtspunkt im Vordergrund steht, möge ein Beispiel aus einer

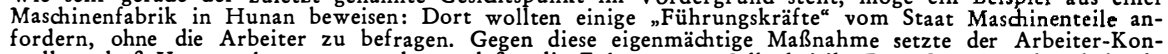
trollausschuß Versammlungen an und veranlaßte die Führung zur Selbstkritik. Dem Staat wurden dadurch, wie es hieß, 250000 Yüan erspart (SWB 3582 B II 1 f.).

63 SWB 3455 B II 3; 3637 B II 14 f.; Volkszeitung 4. 3. 1970.

$64 \mathrm{Vgl}$. hierzu Georg Brunner "Kontrollfunktionen und Kontrollorgane in der Sowjetunion und in Mitteldeutschland“, Bd. 16/67 der „Berichte des Bundesinstituts für ostwissenschaftliche und internationale Studien ${ }^{\star}$, Köln, S. 13 .

65 Zum 'Vorwurf, daß "unzivilisierte Leute“ doch keine Fabrik kontrollieren könnten, vgl. SWB 3455 B II, 3. 66 Gemeint sind offenbar die vom Staat enteigneten und regelmäßig abgefundenen früheren Fabrikbesitzer. 67 SWB 3360 B II 7.

68 SWB 3455 B II 3 .

69 SWB 3360 B II 7: Ihre Aufgabe ist es u. a., den üblichen Vergleich zwischen nvergangenem Elend und gegenwärtigem Glück“ zu ziehen.

70 7. SWB 3455 B II 3.

71 Vgl. z. B. China News Nr. 380 vom 8. 10. 1970.
} 
„Berichtsgruppen“ der Revolutionskomitees üben Kontrolle aus. Die „Berichtsgruppen "72 bestehen aus führenden Funktionären, Freizeitkorrespondenten und ähnlichen Aktivisten. Sie sind verantwortlich für politische Studienkurse und für die korrekte Durchführung der Weisungen ${ }^{73}$. Überwachungsgruppen können auch von außerhalb des Fabrikbetriebes kommen ${ }^{74}$.

Von klaren Kompetenzen - wie etwa in den Fabrikbetrieben der Sowjetunion kann hier also keine Rede sein. Die vielfachen Überschneidungen machen vielmehr deutlich, daß es sich hier nicht um bürokratisch ausgeformte "formelle" Kontrollinstanzen handelt, sondern vielmehr um kaleidoskopartige, "revolutionäre" Entfaltungen der Massen, die meist im Dienste der „inneren Kontrolle“ stehen. Sollten allerdings wirtschaftliche Bestrebungen weiterhin jenen hohen Stellenwert beibehalten, der ihnen seit dem Ende der Kulturrevolution zukommt, so würde äußere Kontrolle wieder stärker in den Vordergrund treten. Massenselbstkontrolle, die allerdings an wichtigen Stellen von Militärkontrolle flankiert ist, ersetzt also weitgehend die früher durch die Polizei ausgeübte Staatskontrolle.

bb) Staatsanwaltschaft ${ }^{75}$ : Der bis zur Kulturrevolution hochzentralisierten Staatsanwaltschaft oblag die allgemeine Aufsicht über die Behörden und Bürger; darüber hinaus hatte sie - neben den Organen der öffentlichen Sicherheit - wichtige Kontrollrechte im Strafermittlungsverfahren. Auch die Gerichtskontrolle gehörte zu ihren Aufgaben. Vor allem hatte die Staatsanwaltschaft an den Voruntersuchungen teilzunehmen, bei den Verhandlungen anwesend zu sein und - wenn nötig - gegen Urteile Rechtsmittel einzulegen. Auch bei der Vollstreckung von Urteilen konnte sie Kontrolle ausüben. Häufig gab es Reibereien mit den Parteiorganen, die sich in die Tätigkeit der Staatsanwaltschaft einmischten.

Während der Kulturrevolution wurde die Staatsanwaltschaft, der der Ruf des Bürokratismus und der engen Verbindung zu Liu Shao-ch'i anhaftete, weitgehend aufgelöst und durch Armeevertreter ersetzt. Schon vorher hatte die Partei versucht, die "Massenlinie“ auch im Bereich der Staatsanwaltschaft stärker zur Auswirkung kommen zu lassen. So sollten die Staatsanwälte z. B. mehr an Ort und Stelle ermitteln und überdies regelmäßig an Produktionsarbeiten teilnehmen.

cc) Die Gerichte ${ }^{76}$ : Nach $\ 3$ des Gerichts-Organisationsgesetzes vom 21.9.1954 behandeln die Gerichte Zivil-, vor allem aber Strafsachen, mit dem Ziel, „das volksdemokratische System zu verteidigen, die öffentliche Ordnung aufrechtzuerhalten, das öffentliche Eigentum zu schützen, die gesetzlichen Rechte der Bürger zu sichern und die erfolgreiche Durchführung des sozialistischen Aufbaus sowie die sozialistische Umwandlung zu gewährleisten ". Gerichtsbarkeit zur Nachprüfung staatlicher Verwaltungsakte ist formal nicht vorgesehen. Kontrolle wird insoweit also nur durch den Staatsrat, durch übergeordnete Verwaltungsorgane und vor allem natürlich auch durch die Partei ausgeübt.

\footnotetext{
72 Solche Berichtsgruppen sind z. B. in allen Fabriken mit über 1000 Arbeitern den jeweiligen politischen Abteilungen der Revolutionskomitees eingepflanzt, so wenigstens in Tsingtao, vgl. China News Nr. 353 .

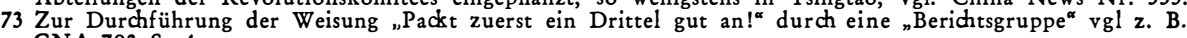
CNA 793 S. 4.

74 Als z. B. eine Großstrickerei in Shao-yang bei der dortigen Volksbank eine Anleihe von 150000 Yüan machen wollte, kam eine Kontrollgruppe des Geldinstituts in die Fabrik, überprüfte deren Bestände und wies nach, daß aufgrund der vorhandenen und ungenützten Ressourcen eine Kreditaufnahme Verschwendung wäre.

75 Genauere Ausführungen über den Aufbau der Staatsanwaltschaft und ihre Aufgaben können hier nicht gebracht werden. Vgl. aber die systematische Darstellung von Shao-chuan Leng "Die Volksstaatsanwaltschaft im kommunistischen China ${ }^{\alpha}$ in "Osteuropa-Recht ${ }^{\alpha}{ }^{1963}$, Heft 3, S. 193-209.

76 Auch hier können keine näheren Ausführungen gebracht werden. Eine systematische Darstellung gibt Taotai Hsia "Die Volksgerichte in der Volksrepublik China“, Osteuropa-Recht 1963, Heft 3, S. $169-192$.
} 
Die offiziellen Presseorgane sind nie müde geworden, besonders die erzieherische Rolle der Gerichte zu betonen. Die Entscheidung ist ein für das Publikum bestimmtes, in populärem Stil gehaltenes Dokument und nimmt damit denselben erzieherischen Stellenwert ein wie etwa ein Drama oder eine Rundfunksendung. Gerichtsverhandlungen werden deshalb nicht selten durch Funk und Fernsehen übertragen. Größere Kriminalfälle sollen in Form von „Massendiskussion und Massenkritik" abgeurteilt werden ${ }^{77}$. Ohnehin sind Gerichtsverhandlungen meist nur e i n Kettenglied innerhalb der jeweils gerade laufenden Massenkampagnen ${ }^{78}$, zu denen "Großversammlungen der Kritik und Verurteilung" sowie Aufmärsche und Ansprachen gehören. Soweit also überhaupt noch Gerichtsverhandlungen abgehalten und nicht etwa durch Kritikversammlungen verdrängt werden, dienen sie dem politischen Anschauungsunterricht. Auch hier ist also die Hinwendung zur inneren Kontrolle unverkennbar.

g) Die vorliegenden Ausführungen wären unvollständig, würde nicht noch ergänzend auf einige juristische Institutionen hingewiesen, von denen staatliche Kontrolle ausgeht.

$\mathrm{Zu}$ erwähnen ist hier etwa das eigenartige chinesische „Vertragssystem“ (ho-t'ung), das - ganz anders als in westlichen Rechtsordnungen - weniger der "legalen" Institutionalisierung und gerichtlichen Erzwingbarkeit schuldrechtlicher Verpflichtungen als vielmehr der politischen Überwachung dient. So werden z. B. „Verträge" zwischen staatlichen Handelsorganisationen und landwirtschaftlichen Erzeugerkollektiven abgeschlossen, um die im staatlichen Wirtschaftsplan nur kursorisch vorgeschriebenen Produktionsziele nach Maßgabe der jeweiligen lokalen Bedingungen zu konkretisieren ${ }^{79}$ und dadurch letzten Endes die bäuerliche Bevölkerung unter Kontrolle zu bringen. Den „Verträgen“ kommt mit anderen Worten nicht, wie im Westen, die Funktion zu, frei abgegebene Willenserklärungen mehrerer Parteien rechtsverbindlich $\mathrm{zu} \mathrm{k}$ o o $\mathrm{rdin}$ i e $\mathrm{ren}$, sondern vielmehr einen Kontrahenten dem anderen nach Maßgabe des jeweiligen staatlichen Wirtschaftsplans zu $s$ u bordin i e r e n. Diese politische Verwendung ${ }^{80}$ des Vertrags - man könnte hier sogar von "diktierten Verträgen" sprechen - bringt es mit sich, daß einerseits von Vertragsfreiheit im westlichen Sinne praktisch nicht die Rede sein $k_{a n n}{ }^{81}$ und daß die Erfüllung des Vertrags andererseits nicht durch eine Drittinstanz, also auch nicht durch ein Gericht erzwungen werden kann ${ }^{82}$. Statt dessen

77 Eine solche Bestimmung findet sich zwar nicht in der Verfassung von 1954, wohl aber in Art. 25 des Entwurfs von 1970: „Ernsthafte konterrevolutionäre und kriminelle Fälle sind in Massendiskussionen und Massenkritiken $\mathrm{zu}$ behandeln

78 Beispiele für Gerichtsversammlungen in diesem Stile bei Weggel „Die chinesischen Revolutionskomitees" a.a.O., S. 79 f. Nicht selten werden solche Gerichtsverhandlungen über Radio und Fernsehen übertragen, ebda; Massenkampagnen, in deren Verlauf es zu besonders zahlreichen Gerichtsverhandlungen kam, waren z. B. die Landreform, die 3-und-5-Anti-Bewegung und neuerdings - seit 1970 - die sogen. $4-$ Antizewegung ${ }^{\alpha}$ (gegen Korruption, Extravaganz, Nepotismus und Diebstahl); ferner die ${ }_{n} 1-$ Schlag-und-3-AntiBewegung “ von 1971 (Sdiläge gegen Konterrevolutionäre, „anti gegen Korruption und Diebstahl, Spekulation und Horten, Extravaganz und Verschwendung), dokumentarisch belegt in Issues and Studies Februar 1971, S. 64 ff.

79 Richard M. Pfeffer „The Institution of Contracts in the Chinese People's Republic (Part I) China Quarterly Nr. 14 (1963), S. 71, 72 f.

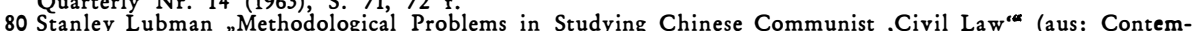
porary Chinese Law: Research Problems and Perspectives, ed. by Jerome Alan Cohen, Cambridge/Mass. $1970)$ S. 245 spricht $\mathrm{m}$. E. etwas zu schwach von "administrativer Verwendung des Vertrages

81 Pfeffer a.a.O. 177.

82 "Gegenwärtig gibt es in unserem Land keine öffentlichen Entscheidungsorgane" (Tuan chi-kuan, vgl. "Chung-hua jen-min kung-ho-kuo min-fa chi-pen wen- $t$ ' $i^{\star}$ (Grundprobleme des bürgerlichen Rechts der Volksrepublik China), hrsg. vom Institut für ziviles Recht der Zentralen Kaderschule für Politik und Recht, Peking 1958. Dieser im Lehrbuchstil aufgebaute Text ist - in Ermangelung eines zivilrechtlichen Gesetzbuches - praktisch dessen Ersatz, oder war es zumindest bis zur Kulturrevolution. Zu großer BeGesetzbuches - praktisch dessen Ersatz, oder war es zumindest bis zur Kulturrevolution. Zu großer Be-
deutung haben es die im Lehrbuch aufgeführten Bestimmungen allerdings nie bringen können, da 1. kaum juristisch ausgebildetes Personal vorhanden war, das die Bestimmungen hätte in die Praxis umsetzen können und da 2. das Regime zu keinem Augenblick geneigt war, sich nach rechtsstaatlichen Gesichtspunkten gesetzlichen Regelungen zu unterwerfen. 
greift bei Verzug, positiver Vertragsverletzung oder Unvermögen „Kontrolle“ (chien-ch'a) durch einen Notar, durch die zuständige Volksbank (Drohung mit Kreditentziehung usw.) oder aber durch übergeordnete Verwaltungsorgane der jeweils betroffenen staatlichen Vertragsstelle ein ${ }^{83}$. Selbstverständlich ist daneben jederzeit Kontrolle durch die zuständigen Parteiorgane möglich. Die schon in der traditionellen chinesischen Rechtsordnung spürbare Tendenz, Gerichte auszuschalten und Streitfälle durch interne Schlichtung zu regeln, wurde durch diese kommunistischen Praktiken noch mehr verstärkt und dadurch „politisiert“, daß „Kritik und Selbstkritik" der für den Vertragsbruch „Verantwortlichen"84 immer stärker in den Vordergrund rücken. Schon in den fünfziger Jahren war es z. B. üblich, bei Vertragsstörungen die verantwortlichen Funktionäre zum „Eingeständnis ihrer Fehler" zu veranlassen ${ }^{85}$. Diese Tendenz hat sich seit der Kulturrevolution noch verstärkt. Nunmehr sind die Revolutionskomitees der staatlichen Handeslorganisationen dazu übergegangen, landwirtschaftliche Kader, die den an sie „vertraglich“ gestellten Erwartungen nicht entsprechen, in sogenannten „Mao-Tse-tung-Studienkursen " zu schulen ${ }^{86}$, und zwar hauptsächlich durch sogenannte „kommerzielle Arbeiter des 7. Mai“" ${ }^{87}$, die zu diesem Zweck "nach unten geschickt" werden ${ }^{88}$.

Diese Hinwendung zur inneren Kontrolle hat ihren guten Grund. Zwar ist in chinesischen Verlautbarungen häufig genug von sogenannten „Fünfjahresplänen“ die Rede. Ob jedoch der dritte $(1966-1970)$ und der seit 1971 laufende vierte Fünfjahresplan etwas anderes als Papiertiger sind, läßt sich aufgrund der vorliegenden Informationen nicht entscheiden. Vergleicht man jedenfalls die Verwirklichung des ersten Fünfjahresplans mit der heutigen offensichtlich ausschließlich „verbalen“ Praxis, bedenkt man weiterhin, daß die Dezentralisierung gerade seit der Kulturrevolution stärker als je um sich greift, und hält man sich schließlich vor Augen, daß verschiedene Provinzen dazu übergegangen sind, ihre eigenen Pläne zu entwerfen ${ }^{89}$, so kann man zumindest an der praktischen Realisierung dieser Fünfjahrespläne seinen Zweifel haben. - Sollte diese Vermutung zutreffen, so wäre den "Verträgen“ von ehedem, die ja in den Fünfjahresplänen wurzelten, der Boden entzogen. Damit aber entfielen wesentliche Voraussetzungen für die bisher geübte äußere Kontrolle durch „Verträge“. Was blieb unter diesen Umständen übrig, als die so entstandene Lücke durch innere Kontrolle zu ersetzen?

Die Liste staatlicher Kontrollinstitutionen ließe sich noch erweitern, beispielsweise durch Darstellung der staatlichen Schlichtungskomitees, der Notariate und ähnlicher Einrichtungen, die alle im Laufe der Zeit zunehmend "entlegalisiert" und immer stärker "politisiert" wurden ${ }^{90}$. Es sollte aber bereits aufgrund der bisherigen Ausführungen deutlich geworden sein, daß all diese Institutionen im Spannungsfeld zweier entgegengesetzter politischer Tendenzen stehen, nämlich der Sta-

\footnotetext{
83 Ebda. 210-212.

84 Der Ausdruck "tse-jen“ (Verantwortlicher) kehrt in den „Verträgen“ immer wieder, so daß Lubman a.a.O. wohl zu Recht darauf hinweist, daß eine wesentliche Funktion der Verträge vor allem darin bestand, die für das Gelingen des Vertrags "Verantwortlichen“ festzulegen.

87 Maos Weisung vom 7. Mai 1966 ist eine Direktive, in der darauf hingewiesen wird, daß aus der Volksbefreiungsarmee, den Fabriken, den Dörfern, den Schulen, den Handelsunternehmungen, den Dienstleistungsbetrieben und den Organen der Partei und des Staates "große Schulen der Revolutionierung " gemacht werden sollen. In dieser Weisung, die ursprünglich in einem Brief Maos an Lin Piao enthalten war, und die zur wohl berühmtesten "Direktive des Vorsitzenden “ während der Kulturrevolution überhaup wurde, ist die maoistische Gesellschaftsstrategie wie in einem Brennsglas eingefangen. Kein Wunder, daß das Epitheton "7. Mai“ im Zusammenhang mit zahlreichen Organisationen auftaucht.

88 SWB 3446 B 9 " f.

89 Vgl. dazu CNA 795 S. 4

90 Vgl. dazu i. ü. Lubmann a.a.O. $233 \mathrm{ff}$.
} 
bilisierung einerseits und der „revolutionären Mobilisierung“ andererseits, und daß sie demzufolge je nach der konkreten historischen Entwicklung bald mehr im Sinne äußerer Kontrolle (im Extremfall: formeller Kontrolle), bald mehr im Sinne der inneren Kontrolle funktionieren.

3. Militärkontrolle: Seit die Partei- und Staatsorganisationen während der Kulturrevolution weitgehend zusammenbrachen, liegt die eigentliche Kontrolle bei der Armee. Daran ändert auch die seit 1969 laufende Bewegung zum Wiederaufbau der Partei wenig, da die neuen Organisationen ja stark mit Militärs durchsetzt sind. Zwei Kontrollbereiche gilt es zu unterscheiden: den Kontrollmechanismus im „besonderen Gewaltverhältnis“ der Volksbefreiungsarmee sowie der paramilitärischen Verbände einerseits und die gegebenen Kontrollmöglichkeiten des Militärs nach außen, also in Richtung auf den „zivilen“ Bereich.

a) Der innermilitärische Kontrollapparat, der in der straffen Hand des militäreigenen Parteiapparats liegt, hat sich als eine der ganz wenigen Organisationsstrukturen in der Volksrepublik China nahezu unversehrt über die Kulturrevolution hinwegretten können. Teils lag dies daran, daß die Kulturrevolution seit 1961 (Lin Piao wurde damals Verteidigungsminister) in der VBA durch forcierte Indoktrinations- und Ausrichtungsbewegungen bereits vorweggenommen worden war, teils ist dies Ursache und Folge der ebensooft wegdiskutierten wie dann doch immer wieder verifizierbaren Tatsache, daß Volksbefreiungsarmee und Volksmassen alles andere als identisch sind.

Die Parteikontrolle in der Armee läuft durch drei Instanzen: An der Spitze steht die "Allgemeine Politische Abteilung “91, die "unter der Anleitung des ZK ${ }^{92}$ die ideologische und organisatorische Arbeit der Partei in der Armee wahrnimmt" ${ }^{\text {"93 }}$. Direktiven der allgemeinen Politischen Abteilung werden durch die Parteiausschüsse auf allen Ebenen an die Armee weitergeleitet, und zwar an die Militärbefehlshaber bzw. an die Politkommissare, zwischen denen ursprünglich eine „Arbeitsteilung " in dem Sinne bestand, daß die Befehlshaber militärische Angelegenheiten auszuführen, die Politkommissare dagegen „politische Arbeit“ zu leisten hatten ${ }^{94}$. Seit mit Beginn der sechziger Jahre die "Politik“ wieder "das Kommando übernommen" hat, gibt es freilich keine rein militärischen Angelegenheiten mehr. Selbst Fragen der Militärtechnologie sind nunmehr durch die „politische“ Brille zu betrachten, mit der Folge, daß der Militärbefehlshaber gegenüber dem Politkommissar immer mehr das Nachsehen hat. Auch in dieser Verschiebung manifestiert sich eine Wendung von der äußeren Kontrolle hin zur inneren Kontrolle: Hatte der Politkommissar vorher die Ausführung durch den Militärbefehlshaber überwacht, so war er nunmehr durch die zunehmende Politisierung sämtlicher Problembereiche gezwungen, die politische Indoktrinierung zu verschärfen.

91 Tsung cheng-chi pu. von "Partei“ die Rede (Art. 9 IV, 5 V).

93 kuan-li; Art. 35 der Parteisatzung von 1956; in Art. 9 Abs. 4 der Parteisatzung von 1969 heißt es: „Unter der Anleitung des Vorsitzenden, des Stellvertretenden Vorsitzenden und des Ständigen Ausschusses des Politbüros des ZK werden einige erforderliche vereinfachte und leistungsfähige Organe geschaffen, die die laufende Arbeit der Partei, der Regierung und der Armee auf zentralisierte Weise erledigen."

94 Ellis Joffe "The Communist Party and the Army “ in "Contemporary China“ 1959/60 ed. by Stuart Kirby, Hong Kong 1961, S. 62 f. ders. "Party and Army, Professionalism and Political Control in the Chinese Officer Corps 1949-1964“, Cambridge/Mass. 1965 (zitiert Joffe 2). 
Kein Zufall ist es, daß diese Wandlung des Kontrollmechanismus begleitet war von vier weiteren auffälligen Phänomenen. - An die Stelle des („unpolitischen“) Professionalismus, der moderne Bewaffnung, technisches Training (Militärakademien), Institutionalisierung (Konskription, militärische Karrieren) und ein Berufsheer befürwortete (Armee als "Gesellschaft" i. S. von Tönnies), trat nach und nach die („politische") Guerillakonzeption, die nach wie vor den „Volkskrieg“ propagierte und auf „Einheit zwischen Offizieren und Mannschaften, zwischen Offizieren und Untergebenen sowie zwischen militärischen, politischen und produktiven Tätigkeiten" Wert legte95 (Armee als "Gemeinschaft"). Auch die damals global bekanntgewordene Aufhebung der Ränge und Abzeichen sowie die Politik des „Hinunterschickens“ der Offiziere gehört in diesen Entwicklungsbereich.

- Vor allem das Verhältnis zwischen Offizieren und Mannschaften erfuhr dadurch eine tiefgreifende Wendung: Anstelle von Subordination, Statusbewußtsein, Distanz und bürokratischem „Zentralismus" sollten Gleichberechtigung (der Offizier hat lediglich mehr Pflichten!), gegenseitige Erziehung ${ }^{96}$, Kameraderie und „Demokratie“ treten: Demokratie vor allem auf den „drei Gebieten"97 der Politik (offene Aussprachen, Kritik und Selbstkritik) ${ }^{93}$, der Wirtschaft (Mitsprache in Verpflegungsfragen u. ä.) und des Militärischen (Diskussion taktischer Fragen vor, während und nach militärischen Operationen $)^{99}$.

- Im Verhältnis zwischen Armee und Volk trat insofern eine Anderung ein, als die alte „Fisch-Wasser-"Theorie nach und nach die Kasernenhofmentalität wieder verdrängte (Parole: „Hilf der Armee, liebe das Volk“).

- Die Renaissance der "Politik" in der Armee wirkte sich schließlich auch auf das Verhältnis zwischen militärischem und nichtmilitärischem Einsatz aus. Hatte die VBA eine Zeitlang nichtmilitärische Arbeit nur zum Zweck ihrer militärischen Vervollkommnung begriffen, so oblag es ihr von nun an, sich nicht mehr nur als „Kampftruppe“ zu empfinden, sondern ebensosehr als Produktionstruppe (Neulanderschließung, Ernteeinsätze, Selbstversorgung) und als Propagandatruppe für die Volksmassen (VBA als politisches Modell für die ganze chinesische Gesellschaft).

Je stärker sich also die Armee „repolitisierte“ und damit „revolutionierte“, um so deutlicher trat die innere Kontrolle in den Vordergrund.

b) Von der innermilitärischen Kontrolle ist die Überwachung der „Volksmassen“ durch die Armee zu unterscheiden. Seit die „zivilen“ Parteiorganisationen während der Kulturrevolution weitgehend zusammenbrachen, wuchsen die wichtigsten Kontrollkompetenzen de facto nach und nach der Armee zu, obwohl seit 1969 der Parteiapparat langsam wieder aufgebaut wird.

Drei Kontrollorganisationen haben sich in diesem Zusammenhang seit der Kulturrevolution herausgebildet ${ }^{100}$.

\footnotetext{
$95 \mathrm{Zu}$ dieser „Einheit nach innen“ vgl. Mao Tse-tung „Uber die Koalitionsregierung ${ }^{*}$, Ausgewählte Werke Bd. 3 Peking 1969, S. 250.

96 Worte des Vorsitzenden Mao a.a.O. 199

97 San ta min-chu („Drei große Demokratien “).

98 Worte des Vorsitzenden Mao a.a.O. 192, Mao Tse-tung „Der Kampf im Djinggang-Gebirge“, Ausgewählte Werke Bd. 1, Peking 1968, S. 91.

$99 \mathrm{Mao}$ Tse-tung „Die demokratische Bewegung in der Armee*, Ausgewählte Werke, Bd. 4, Peking 1969, S. $199 \mathrm{f}$.

100 Eine systematische Darstellung zu dieser Frage in Oskar Weggel „The PLA in the Cultural Revolution, Grass-Root Level Organization and Mass Work “ in Revue du Sudest-Asiatique et de l'Extrême Orient 1969, S. 243 bis 254 .
} 
aa) Sogen. „Militär-Kontrollausschüsse“101: Es sind dies Übergangsorganisationen, die meist durch die später entstehenden Revolutionskomitees in den betreffenden Einheiten aufgesogen werden. Militär-Kontrollausschüsse in den Ämtern für öffentliche Sicherheit übernehmen Gerichtsfunktionen und haben in dieser Eigenschaft sogar schon Todesurteile gefällt. Diese Komitees sind eine organisatorische Reminiszenz an jene Militär-Kontrollausschüsse, die 1949 bei der Eroberung des Festlandes von den vorstoßenden VBA-Einheiten jeweils sofort nach der „Befreiung“ einer Stadt oder eines Kreises eingesetzt wurden. Die Militär-Kontrollausschüsse organisierten damals sogenannte „Übernahmekomitees“, die Universitäten, Schulen und Verlage besetzten (kulturelle Übernahmekomitees), Banken, Fabriken und Warenhäuser der Kuomintang übernahmen (wirtschaftliche Übernahmekomitees), militärische Einrichtungen in die Hand nahmen (militärische Übernahmekomitees) und lokale sowie zentrale Behördenstellen ablösten (administrative Übernahmekomitees) ${ }^{102}$. Aufgabe der Militär-Kontrollausschüsse war es, für die Disziplin der eigenen Soldaten bei der Übernahme zu sorgen, Massengerichtsverfahren zu organisieren, kämpfende Truppen sowie Verwaltungspersonal der Kuomintang umzufunktionieren und dadurch die Ausführung von Weisungen der Parteileitung bei der Übernahme von Kuomintang-Gebieten zu gewährleisten. Die Militär-Kontrollausschüsse übten diese Funktion bis etwa 1951 aus, waren also nur vorübergehend im Amt. Nach Ausbruch der Kulturrevolution, vor allem seit 1967, herrschten in den chinesischen Großstädten oft ähnlich ungeordnete Verhältnisse wie 1949: Rundfunkanstalten, Verlage, Hochschulen, ja Ministerien mußten „besetzt“ und übernommen werden. $\mathrm{Da}$ die Rotgardisten bei solchen Aktionen Mangel an Disziplin zeigten, traten Militär-Kontrollausschüsse an ihre Stelle. Sie leiteten das besetzte Unternehmen, „behandelten“ Straftaten, wobei Todesurteile keine Seltenheit waren ${ }^{103}$, gaben Erlasse heraus ${ }^{104}$, überprüften Namenslisten ${ }^{105}$ und taten alles in ihrem Ermessen Stehende, um die „Diktatur des Proletariats zu konsolidieren, die Konterrevolutionäre zu unterdrücken und die Kulturrevolution zu verteidigen"106. Kontrollorgane - und nicht etwa reine Führungsorgane - waren die Militär-Kontrollausschüsse insofern, als sie die Durchführung der - freilich äußerst weitherzig gefaßten - „Weisungen“ Mao Tse-tungs bzw. des Zentralkomitees und des Staatsrats zu überwachen hatten ${ }^{107}$. Wie schon nach 1949 waren die Militär-Kontrollausschüsse auch in der Kulturrevolution nur mit vorübergehenden Aufgaben betraut. Nach und nach wurden - und werden - sie von den neu entstehenden Revolutionskomitees und Parteiausschüssen abgelöst.

bb) Die wichtigste Kontrollfunktion kommt heutzutage den sogenannten „lokalen Truppen zur Unterstützung der Linken" ${ }^{108} \mathrm{zu}$, die in den Revolutionskomitees und, seit Beginn der Bewegung zum Wiederaufbau der Partei, auch in den Parteiausschüssen nach dem Schema der Drittelparität neben den Massenvertretern und Funktionären mitwirken. Auch hier hat sich das Gesetz der zunehmenden Dichte erneut bestätigt: In den Revolutionskomitees auf Provinzebene rekrutierten sich

101 Chün-shih kuan-chih wei-yüan-hui.

102 Vgl. dazu John Gittings "The Role of the Chinese Army*, London-New York-Toronto 1967, S. 265 ff.

103 Vgl. dazu Weggel Revolutionskomitees a.a.O. S. $79 \mathrm{f}$.

104 Vgl. z. B. CEP Documents of the Great Proletarian Cultural Revolution a.a.O., S. $674,680 \mathrm{ff} ., 686$.

105 Survey of China Mainland Press (SCMP) Nr. 4130, S. 15.

106 CCP Documents of the Great Proletarian Cultural Revolution a.a.O., S. 667.

107 Näheres zur "Gesetzgebungstechnik “ und zu den weiten Rahmenbestimmungen vgl. Weggel, Gesetzgebung a.a.O., S. $147^{\prime} \mathrm{ff}$. und $152 \mathrm{f}$.

108 dih tso hsiao-tsu. 
im Jahre 1970 nicht weniger als 21 von 29 Ersten Vorsitzenden sowie 72 von 216 Vorstandsmitgliedern aus den Reihen der Volksbefreiungsarmee. Ein ähnliches Bild ergibt sich bei den neuen Parteiausschüssen auf Provinzebene. Wo untergeordnete Revolutionskomitees oder Parteiausschüsse $\mathrm{zu}$ besetzen sind, werden nicht reguläre Armeevertreter, sondern Milizionäre eingesetzt, die als eine Art verlängerter Arm der Volksbefreiungsarmee gelten dürfen.

cc) Als weitere Kontrollorganisationen haben sich die „Zentralen Truppen zur Unterstützung der Linken" bewährt, die als eine Art Feuerwehr überall dort eingreifen, wo die lokalen "Hilfe-links“-Aktionen versagen ${ }^{109}$. Unter dem Legitimationstitel der sogenannten „Drei Unterstützungen und zwei Militärischen“ (Funktionen, d. h. Unterstützung der Linken, der Arbeiter und der Bauern, sowie Militärkontrolle und Militärausbildung) ${ }^{\mathbf{1 1 0}}$ kontrolliert das Militär mit anderen Worten direkt durch die Kontrollausschüsse und indirekt durch die Revolutionskomitees bzw. die neuen Parteiausschüsse. Überdies ist die Armee zum Vorbild im „lebendigen Studium und in der lebendigen Anwendung der Mao-Tse-tungIdeen" geworden, nimmt also nun auch im Bereich der inneren Kontrolle eine führende Position ein. Auch die Militarisierung der Sprache hat das Denken der Volksmassen für die Propaganda griffiger gemacht ${ }^{111}$.

Einem Mißverständnis ist hier noch vorzubeugen: Vor allem seit der Kulturrevolution ist es üblich geworden, von "Militärherrschaft" in China zu sprechen, wobei die Ausdrucksweise nahelegt, daß hiermit nicht nur Ausführung oder Kontrolle, sondern „Führung“ gemeint ist. Zwei Einschränkungen sind hierzu angebracht:

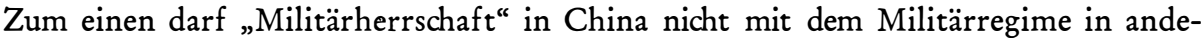
ren Ländern der Dritten Welt verglichen werden; denn die Verbindung mit den Volksmassen ist, wie oben ausgeführt, im Zeichen der Repolitisierung wieder enger geworden, so daß in China eher als in anderen Ländern von einer Volksbefreiungsarmee gesprochen werden darf. Zum anderen sollte man nicht von einer $\mathrm{F} \ddot{\mathrm{h}} \mathrm{r}$ u n g s rolle der Armee sprechen, wenngleich nicht zu leugnen ist, daß der Anteil des Militärs in den Revolutionskomitees und in den neu gegründeten Parteiausschüssen auf Provinzebene sowie in den Pekinger Parteispitzengremien beachtlich ist ${ }^{112}$. Deswegen aber schon von Führung $\mathrm{zu}$ sprechen, ginge $\mathrm{zu}$ weit. Soweit die Armee als Vorbild im „lebendigen Studium und in der lebendigen Anwendung der Mao-Tse-tung-Ideen" wirkt und damit die innere Kontrolle mitbestimmt, handelt sie nicht aus eigener Autorität heraus, sondern wirkt - wie es immer wieder heißt - im Geiste Mao Tse-tungs und seines „Hauptquartiers“, wenngleich zugegeben ist, daß zahlreiche Indoktrinationsmethoden (sogen. „3- und 8-Arbeitsstil“, „4-5-Gut-Bewegung“, „4 Erste“, „Rotes Buch“, das von der Armee zusammengestellt wurde usw.) ursprünglich auf die Armee beschränkt war.

109 Vgl. dazu Näheres in Facts and Features, Taipei 18. 9. 1968, S. 20 ff.

110 San chih liang chün.

111 Näheres dazu Weggel, Massenkommunikation a.a.O., S. $39 \mathrm{ff}$., insbesondere S. $44 \mathrm{f}$

112 In den Revolutionskomitees auf Provinzebene rekrutierten sich im Jahre 1970 nicht weniger als 21 von 29 Ersten Vorsitzenden sowie 73 von 216 Mitgliedern aus den Reihen der Volksbefreiungsarmee! Auch die zentrale Parteiapparatur ist stark mit militärischen Elementen durchsetzt. Von den 154 Mitgliedern des IX. ZK, deren Biographien eine Wertung erlauben, durchliefen 55 eine zivile und 99 Personen eine militärische Karriere. Gegenwärtig sind nicht weniger als $45 \%$ im militärischen Sektor tätig. Ahnlich liegen tärische Karriere. Gegenwärtig sind nicht weniger als $45 \%$ im militärischen Sektor tätig. Ahnlich liegen
die Verhältnisse im Politbüro: Von den 17 Vollmitgliedern sind 10 im zivilen Bereich und 7 im Militärapparat tätig; zu den Parteizahlen vgl. Erik v. Groeling „Innenpolitik und Organisation im kommunistischen China ${ }^{\alpha}$, Schriftenreihe der niedersächsischen Landeszentrale für politische Bildung, Hannover 1971, S. 95 und 113; der zahlenmäßige Anteil des Militärs an den neu errichteten Parteiausschüssen auf Provinzebene war zum Zeitpunkt der Abfassung des vorliegenden Aufsatzes noch nicht zu ermitteln. Wollte man jedoch einen Schluß von den anfänglich gegründeten Parteiausschüssen auf alle weiteren ziehen, so entspricht der Anteil des Militärs an den Parteiausschüssen dem Anteil an den Revolutionskomitees auf Provinzebene. 
Soweit die Armee für die Durchführung politischer Beschlüsse sorgt, übt sie Ausführungskontrolle. Solche Beschlüsse, ob sie nun in Form von „Verwaltungsakten“ oder von „Rechtsnormen“ ergehen, werden nicht von der VBA gefaßt, sondern von Gremien, in denen die VBA zwar vertreten ist, aber kein Monopol innehat. Dies galt mit Sicherheit bis zur Kulturrevolution. Während der Kulturrevolution waren es vor allem Weisungen des ZK, des Militärausschusses beim ZK, des Revolutionsausschusses beim ZK und des Staatsrats bzw. sogenannte "Neueste Weisungen des Vorsitzenden Mao", deren Ausführung es zu überwachen galt. Aber auch nach der Kulturrevolution ist die VBA keineswegs identisch mit der Führung. Höchste Autorität kommt nach wie vor dem Ständigen Ausschuß beim Politbüro zu. Neben dem Politbüro und dem ZK spielen vor allem einige informelle Gremien wie die „Zentrale Arbeitskonferenz“, Treffen von Parteiführern auf Provinzebene, „Adhoc-Parteikonferenzen" und ähnliche Organisationen eine wichtige Rolle im Entscheidungsprozeß113. In all diesen Versammlungen ist die VBA zwar von großer, aber nicht von ausschlaggebender Bedeutung. Insofern bleibt sie trotz ihres immer stärker werdenden Einflusses im wesentlichen doch auf Kontrolle beschränkt.

\section{Zusammenfassung}

Auf das politische System der Volksrepublik China paßt weder das westliche DreiGewalten-Schema noch die Fünf-Gewaltenlehre, wie sie z. B. in die Verfassung der Republik China Eingang gefunden hat. Vielmehr empiehlt sich die wesentlich pauschalere Einteilung in Führung, Ausführung und Kontrolle. Kontrolle ist hierbei ein System der Beaufsichtigung, dessen Zweck es ist, ex ante oder aber ex post die Ausführung von Weisungen und politischen Richtlinien („Ausführungskontrolle"), aber auch umgekehrt die Führungsorgane zu überwachen („Führungskontrolle"), wobei Indoktrinisierungsmaßnahmen im weitesten Sinne („innere Kontrolle") oder aber organisatorisch verselbständigte Apparate eingesetzt werden („äußere Kontrolle“), die ihrerseits ausdrücklich für Kontrollzwecke vorgesehen sind („formelle Kontrolle") oder aber Kontrollbefugnisse implizit neben anderen Aufgaben wahrnehmen („informelle Kontrolle"). Wichtig ist hierbei vor allem die „innere Kontrolle“, die in den Bezugsrahmen der "Sozialkontrolle" gehört und in dieser Eigenschaft als ein System prophylaktischer Überwachung durch permanente Indoktrination funktioniert. Dabei spielen Techniken der Massenkommunikation eine wesentlich größere Rolle als Administration und äußere Machtanwendung. Statt von innerer Kontrolle könnte man auch von „Steuerung “ sprechen.

Die Kontrollpolitik ist sich im Verlauf der Geschichte der Volksrepublik China nicht immer gleich geblieben. Herrschte in der ersten Hälfte der fünfziger Jahre unter dem Einfluß des sowjetrussischen Modells noch vielfach das Prinzip der äußeren Kontrolle vor, das zumeist mit Ausführungs- und Ex-post-Kontrolle identisch war, so verlagerte sich - entsprechend dem alten Grundthema der „Massenlinie" - das Schwergewicht seit dem 8. Parteitag (1956) zunehmend auf die innere Kontrolle. Kritik und Selbstkritik, Plakat- und Briefaktionen, „Hinunterschicken“ und intensive Gruppenarbeit sollten fortan den formalen und bürokratisierten Inspektionsmechanismus überflüssig machen.

113 Näheres hierzu Parris H. Chang "Research Notes on the Changing Loci of Decision in the CCP“, China Quarterly Nr. 44, S. $169 \mathrm{ff}$. 
Die Verflochtenheit von Partei, Staatsapparat, Militär und Massenorganisationen macht es schwierig, spezifische Kontrollformen aus dem Gesamtmechanismus auszusondern. Trotzdem kann - cum grano salis - von Parteikontrolle, Staatskontrolle und Militärkontrolle die Rede sein. Die Partei kontrolliert in dreifacher Weise: durch Überwachung sämtlicher Administrativorgane, Beaufsichtigung aller kultureller pädagogischer und wirtschaftlicher Einheiten („Parteizellen“) und durch ständige Überprüfung ihrer eigenen Mitglieder, wobei sogen. „kleine Studiengruppen“, sorgfältig geführte Personalakten, sogen. „Arbeitsausschüsse“ und vor allem regelmäßige "Ausrichtungsbewegungen" eine große Rolle spielen. Mit der Partei aufs engste verbunden sind auch die Massenorganisationen, vor allem die Kommunistische Jugendliga.

Im Bereich der Staatskontrolle spielten neben den zentralen und lokalen Organen vor allem Polizei, Staatsanwaltschaft und Gerichte eine besonders wichtige Rolle. Durch die Kulturrevolution wurde der gesamte Sicherheitsapparat zerstört oder von der Volksbefreiungsarmee übernommen und seine Funktionäre in sogenannte „Arbeitsschulen des 7. Mai“ zur Umerziehung geschickt. Die Sicherheitskompetenzen gingen auf die während der Kulturrevolution gegründeten Revolutionskomitees über, die nach und nach ein buntgewebtes Netz von Sicherheitsorganisationen aufbauten, das nicht mehr den einheitlichen Charakter von früher trägt. Nicht zuletzt hängt diese Entwicklung mit der Internalisierung der Kontrolle zusammen, die vor allem in den überall aus dem Boden schießenden „Mao-Tse-tung-Studienklassen " ihren Ausdruck findet. Staatliche Kontrolle geht auch von dem eigenartigen chinesischen System „zivilrechtlicher Verträge“ aus, das weniger der legalen Institutionalisierung und gerichtlichen Erzwingbarkeit schuldrechtlicher Verpflichtungen als vielmehr der politischen Überwachung dient.

Seit die Partei- und Staatsorganisation während der Kulturrevolution weitgehend zusammenbrachen, kommt vor allem der Militärkontrolle überragende Bedeutung zu. $\mathrm{Zu}$ unterscheiden ist hier die innermilitärische Kontrolle, die in der straffen Hand des militäreigenen Parteiapparats liegt und die Überwachung der „Volksmassen" durch die Armee. Drei Kontrollorganisationen haben sich seit der Kulturrevolution vor allem herausgebildet, nämlich die sogen. „Militärkontrollausschüsse“, die sogen. „lokalen Truppen zur Unterstützung der Linken, der Industrie und der Landwirtschaft" sowie die „zentralen Unterstützungstruppen“. Unter dem Legitimationstitel der sogen. „drei Unterstützungen und zwei Militärischen " (Unterstützung der Linken, der Arbeiter und Bauern, Militärkontrolle und Militärtraining) kontrolliert das Militär direkt durch die Kontrollausschüsse und indirekt durch die Revolutionskomitees bzw. die neuen Parteiausschüsse, in die Militärvertreter entsandt werden. Úberdies ist die Armee zum Vorbild im „lebendigen Studium und in der lebendigen Anwendung der Mao-Tse-tung-Ideen" geworden, nimmt also nun auch im Bereich der inneren Kontrolle eine führende Position ein. 